\title{
Improved QO-STBC OFDM System Using Null Interference Elimination
}

\author{
K. O. O. Anoh, R. A. Abd-Alhameed, Y. A. S. Dama, S. M. R. Jones, T. S. Ghazaany, J. Rodrigues and K. N. \\ Voudouris \\ Mobile and Satellite Communications Research Centre, University of Bradford, UK BD7 1DP
}

\begin{abstract}
The quasi-orthogonal space time block coding (QO-STBC) over orthogonal frequency division multiplexing (OFDM) is investigated. Traditionally, QO-STBC does not achieve full diversity since the detection matrix of QO-STBC scheme is not a diagonal matrix. In STBC, the decoding matrix is a diagonal matrix which enables linear decoding whereas the decoding matrix in traditional QO-STBC does not enable linear decoding. In this paper it is shown that there are some interfering terms in terms of non-diagonal elements that result from the decoding process which limit the linear decoding. As a result, interference from the application of the QO-STBC decoding matrix depletes the performance of the scheme such that full diversity is not attained. A method of eliminating this interference in QO-STBC is investigated by nulling the interfering terms towards full diversity for an OFDM system. It was found that the interference reduction technique permits circa 2dB BER performance gain in QO-STBC. The theoretical and simulation results are presented, for both traditional QOSTBC and interference-free QO-STBC applying OFDM.
\end{abstract}

Keywords-QO-STBC; STBC; OFDM; Decoding matrix; NullInterference; Interference;

\section{INTRODUCTION}

Present days wireless communications systems are in great quest for efficient communications. Wi-Fi and terrestrial base stations are increasingly deploying the multi-antenna system for seamless communications. For instance, the multiple input multiple output (MIMO) antenna configuration is useful in achieving higher throughput in these wireless communication systems. Space Time block coding (STBC) is one of interesting methods for deploying this technique. The advantage of using, for example, the orthogonal STBC (OSTBC) is that it exploits full power transmission for orthogonal codes so long as the transmitter diversity order is no more than two [1-3]. For more than two transmit diversity, it has been shown that full rate power is not possible [4]. Meanwhile, it is possible to deploy the STBC technology in way that full rate power transmission can be achieved. In such case, the codes are rather formed in a special orthogonal way. This is usually discussed as the quasi-orthogonal STBC, hereinafter QO-STBC. The QO-STBC offers the advantage of improved channel capacity and also improved bit error ratio (BER) statistics for a multi-antenna transmission [2].

In $[1,5,6]$, a QO-STBC was introduced. It achieves full transmission rate but not full diversity [7]. QO-STBC sacrifices both BER measure with increasing SNR and full transmission diversity but offers excellent transmission rate. The BER curves suggest that the codes outperform the codes of orthogonal design only at low SNRs, but worsen at increased SNRs [1]. This is due to the fact that the slope of the performance curve depends on the diversity order gain. One of the major problems that limits the BER performance of the QO-STBC system is from the interference incurred in the decoding process. If these interferences can be removed, then the performance of the QO-STBC scheme will improve towards full diversity gain.

An example of the approach deployed towards achieving full diversity by interference reduction has been shown in [2, 8]. The method involves nulling the interfering terms in the resulting decoding process to improve the performance of the scheme. Then, it is well known that combining OFDM with MIMO thrives towards achieving improved BER and better throughput [9]. The OFDM treats frequency selective channel as flat fading channel with cyclic prefix (CP) that is at least equal to the channel delay spread. This is used to overcome inter-symbol interference (ISI). In this study, the channel model is limited to a correlated multipath transmission only. In such case, the design exploits multipath channel gains due to multipath of flat fading transmission or frequency nonselective channel. The individual path gains for a particular transmission branch are assumed to be uniform.

In this study, the QO-STBC method is studied for three and four transmit antennas. Using the interference elimination approach, it will be shown that the behaviour of QO-STBC can be improved.

The OFDM system and channel model are discussed in Section II and the full transmission rate QO-STBC is discussed in Section III. In Section IV, the free interference QO-STBC with full diversity while OFDM based QO-STBC architecture is presented in Section V. The numerical simulation results for QO-STBC OFDM system is discussed in Section VI followed by summarized conclusion.

\section{The Ofdm System AND CHANNEL MODEL}

OFDM divides wideband into many narrow-bands and treat the channel as a flat fading channel. In this section, both the OFDM scheme and the channel model are presented.

\section{A. Orthogonal Frequency Division Multiplexing (OFDM) Technology}

In the baseband of multicarrier system, OFDM multiplexing scheme is used to divide a selected wideband spectrum into many smaller narrow bands. This is achieved using the fast Fourier transform (FFT). Over a frequency selective channel, a predefined length of the symbol is used to 
overcome inter-symbol interference (ISI) for channel impulses with long delay. This symbol length, usually called the cyclic prefix (CP) is usually longer (or least equal to) the length of the worst delay in time. In time domain, an $N$-point FFT OFDM system can be defined as:

$$
b[n]=\frac{1}{\sqrt{N}} \sum_{k=0}^{N-1} B_{(k, l)} e^{j 2 \pi n k / N}, \quad n=0,1,2, \ldots, N-1
$$

In Equation $1, N$ is the number of narrowband subchannels, $1 / \sqrt{N}$ is a scaling factor with $n$ as the index of the prevalent subcarrier. $B_{(k, l)}$ is the $k^{\text {th }}$ sub-channel input symbol of the $l^{\text {th }}$ - constellation mapped using, for instance, QPSK. Meanwhile, the number of FFT points adopted in the design of any specific OFDM structure provides the number of narrowband sub-channels over which the input symbols are multiplexed. Since the design of QO-STBC requires at least 4 constellations, then the QPSK is applied in this study. Considering the $\mathrm{CP}$, Equation 1 is modified to include the guard length as;

$$
b[n]=\sqrt{\frac{N}{N+N_{g}}} \sum_{k=0}^{N-1} B_{(k, l)} e^{j 2 \pi n k / N},-N_{g} \leq n<N
$$

Where $N_{g}$ is the pre-appended cyclic prefix length. This $\mathrm{CP}$ is used to combat inter-symbol interference (ISI) which is caused by multipath delay.

\section{B. Channel Model}

The channel model of QO-STBC involves $N_{\mathrm{T}}$-transmit and $N_{\mathrm{R}}$-receive antennas. The channel is a typical correlated (nonfrequency selective fading) multipath channel with $L$ independent propagation paths and the same power-delay profile. If $h_{i, k}(n)$ represent the channel impulse response, there will be a vector of $\left[h_{i, k}(0) \quad h_{i, k}(1) \quad h_{i, k}(2) \ldots h_{i, k}(L-1)\right] \in C^{1 \times L}$ independent channel taps corresponding to $i^{\text {th }}$-receive antenna and $k^{\text {th }}$-transmit antenna, where $C^{1 \times L}$ is a complex vector. Each of the taps is generally represented according to [10] thus;

$$
h_{i, k}(n)=\sum_{l=0}^{L-1} \alpha_{i, k}(n) \delta\left(t-\tau_{l}\right) e^{j \theta_{l}}
$$

Where $\alpha$ is the gain, $\theta=(2 \pi \ln / N)$ is the phase and $\tau$ the path delay corresponding to $l^{\text {th }}$-path. $0 \leq n \leq N$-1 characterizes the independent OFDM frequency subcarriers. The channel impulse response of Equation 3 for each path is a zero mean complex Gaussian random variable with a normalized variance of unity. For QO-STBC, the codewords are modulated independently for each transmission branch. For instance, if there are four different time slot codewords then there must be four independent OFDM modulators, one for each. For maximum diversity gains, it is required that number of OFDM subcarriers be more than or equal to the number of independent delay paths, $L$ [11]. Now, if $\boldsymbol{C}$ is the independent codeword resulting in the discrete $b_{j}[n]$-term after OFDM modulation, then the received message will be:

$$
r_{k}^{t}(n)=\sum_{i=1}^{N_{T}} b_{j}^{t}(n) H_{i k}^{t}(n)+Z_{k}(n), \quad 0 \leq n \leq N
$$

Notice that $H_{i k}^{t}(n)$ is the channel transfer function (frequency response) which can be represented as;

$$
H_{i k}^{t}(n)=\sum_{l=0}^{L-1} h_{i, k}^{t}(n) e^{-j 2 \pi l n / N}, \quad j=\sqrt{-1}, 0 \leq n \leq N
$$

In Equation $4, Z_{k}(n)$ is the Gaussian noise term of the $n^{\text {th }}$ OFDM subcarrier at $t^{\text {th }}$ OFDM duration. The channel model is assumed to be frequency non-selective across all frequencies.

\section{CONVENTIONAL QO-STBC With Full RATE}

The well-known conventional QO-STBC with full transmission rate for three antennas or more $[1,6]$ encode matrix columns by dividing them into two orthogonal groups. The codes in the columns within each group are not orthogonal but the codes from columns of different groups are orthogonal. This scheme does not achieve full diversity due to some coupling terms between the estimated symbols [8]. The coupling terms are the problems that deplete the BER performance of the QO-OSTBC scheme. However, using the interference mitigation technique reported in $[2,8]$, improved BER performance can be achieved without losing the full rate transmission and diversity. Meanwhile, let the conventional QO-STBC defined in [6] be expressed as:

$$
C=\left[\begin{array}{ll}
\Omega_{12} & \Omega_{34} \\
\Omega_{34}^{*} & \Omega_{12}^{*}
\end{array}\right]=\left[\begin{array}{cccc}
c_{1} & c_{2} & c_{3} & c_{4} \\
-c_{2}^{*} & c_{1}^{*} & -c_{4}^{*} & c_{3}^{*} \\
c_{3} & c_{4} & c_{1} & c_{2} \\
-c_{4}^{*} & c_{3}^{*} & -c_{2}^{*} & c_{1}^{*}
\end{array}\right]
$$

where $\Omega$ represents the traditional Alamouti orthogonal space time block codes [4] defined as:

$$
\Omega_{12}=\left[\begin{array}{cc}
c_{1} & c_{2} \\
-c_{2}^{*} & c_{1}^{*}
\end{array}\right] \text { and } \Omega_{34}=\left[\begin{array}{cc}
c_{3} & c_{4} \\
-c_{4}^{*} & c_{3}^{*}
\end{array}\right]
$$

Equation 6 constructs a code of $P$ constellations transmitted at $T$ time slots. Here, there are 4 constellations and 4 time slots so that the achieved transmission rate $R=1$ (full rate) where $R=P / T$. It must be emphasized that the entries $\left[c_{1}\right.$, $c_{2}, c_{3}, c_{4}$ ] of the matrix $\boldsymbol{C}$ are related to $b$-terms by the OFDM transformation and should not be confused. However, the QPSK mapping scheme is applied in this study.

Following the tradition in $[3,12]$, the equivalent virtual channel matrix following Equation 6 can be defined as:

$$
H_{v}=\left[\begin{array}{cccc}
h_{1} & h_{2} & h_{3} & h_{4} \\
h_{2}^{*} & -h_{1}^{*} & h_{4}^{*} & -h_{3}^{*} \\
h_{3} & h_{4} & h_{1} & h_{2} \\
h_{4}^{*} & -h_{3}^{*} & h_{2}^{*} & -h_{1}^{*}
\end{array}\right]
$$

Let there be $N_{R}$ maximum receive antennas and $N_{T}$ maximum transmit antennas (It is assumed $N_{T}=4$ in this 
study). Then, the received signal at $t$ time slot and on $i$-receive antenna $\left(1 \leq i \leq N_{R}\right)$ can be expressed in a simplified form as:

$$
r_{t, i}=\sum_{l=0}^{L-1} \alpha_{l, i} b_{t, l}+Z_{t, i}
$$

$\alpha_{n, l}$ is the path gain of the $l^{\text {th }}$ path and $Z_{t, i}$ is the additive white Gaussian noise (AWGN) and necessarily a matrix (vector) of the form:

$$
Z_{t, i}=\left[\begin{array}{c}
z_{1} \\
-z_{2}^{*} \\
z_{2} \\
z_{1}^{*}
\end{array}\right]
$$

The path gains are contributed from the impulse responses of the channel individually obtained as in Equation 3. Notice that $h_{i, k}(n)$ belongs to the entries of the virtual channel matrix, $H_{v}$ of Equation 8. This study is a case of a flat-fading transmission with the impulse responses of the channel individual path being correlated, hence,

$$
r_{t, l}=H_{v} b+Z
$$

Equation 11 represents the received symbols at $t^{\text {th }}$-time slot on $i^{\text {th }}$ - receive antenna of the receiver. Now, let the decoding method proceed as:

$$
\hat{b}=H_{v}^{H} \cdot r=H_{v}^{H} H_{v} \cdot b+H_{v}^{H} \cdot Z
$$

Or,

$$
\hat{b}=D_{4} \cdot b+H_{v}^{H} \cdot Z
$$

Where $D_{4}$ is the quasi-orthogonal detection matrix for four transmit antennas and $H_{v}^{H}$ is the Hermitian equivalent of the matrix $H_{v}$. This conventional quasi-orthogonal detection matrix is defined as:

$$
D_{4}=\left[\begin{array}{llll}
\lambda & 0 & \beta & 0 \\
0 & \lambda & 0 & \beta \\
\beta & 0 & \lambda & 0 \\
0 & \beta & 0 & \lambda
\end{array}\right]
$$

Equation 13 represents the estimate of the codes that are inseparable, one from another, and the interference terms also. In orthogonal STBC, the detection matrix, $D$, is always a diagonal matrix, and so enables a simple linear decoding [13]. This is not possible in QO-STBC since the resulting detection matrix (for instance, Equation 13) is not orthogonal and so not a diagonal matrix, instead quasi-orthogonal. Therefore the simple linear decoding cannot be implemented. Thus, an interference free quasi-orthogonal detection matrix must be of the form $[2,8]$ :

$$
D=\left[\begin{array}{cccc}
\lambda+\beta & 0 & 0 & 0 \\
0 & \lambda+\beta & 0 & 0 \\
0 & 0 & \lambda-\beta & 0 \\
0 & 0 & 0 & \lambda-\beta
\end{array}\right]
$$

Where $\lambda$ is the diagonal of the $(4 \times 4) I_{4}$ matrix. This is the sum of the channel power (or the path gains) and represented as $\lambda=\sum_{n=1}^{N}\left\|h_{n}\right\|^{2}, \quad \forall n=0,1,2,3,4$. Also, $\beta$ represents the interfering terms that deplete the full diversity performance expected of the 4-transmit antenna elements and is computed as: $\beta=h_{1} h_{3}^{*}+h_{2} h_{4}^{*}+h_{1}^{*} h_{3}+h_{2}^{*} h_{4}$.

Thus, $\beta$ will degrade the BER performance of the system as long as the aforementioned decoding approach is followed. This can be improved by using a more complex decoding approach such that the better estimate of the transmit symbol be obtained $[8,13]$. Let the effective estimate of the transmit symbols be:

$$
\begin{aligned}
\breve{b} & =\left(H_{v}^{H} \cdot H_{v}\right)^{-1} H_{v}^{H} \cdot r \\
& =\left(H_{v}^{H} \cdot H_{v}\right)^{-1} D_{4} \cdot b+\left(H_{v}^{H} \cdot H_{v}\right)^{-1} H_{v}^{H} \cdot Z \\
& =\left(H_{v}^{H} \cdot H_{v}\right)^{-1} H_{v}^{H} H_{v} \cdot b+\left(H_{v}^{H} \cdot H_{v}\right)^{-1} H_{v}^{H} \cdot Z
\end{aligned}
$$

So that,

$$
\breve{b}=b+\left(H_{v}^{H} \cdot H_{v}\right)^{-1} H_{v}^{H} \cdot Z
$$

$\breve{b}$ is the effective received symbol after channel compensation. This symbol contains the interfering terms which deplete the BER performance. In the next section, a method of eliminating the interference term discussed in $[2,8]$ is discussed.

\section{FREE-INTERFERENCE QO-STBC WITH FULL DIVERSITY}

In this section, the approach for reducing the coupling (interfering) terms in the decoding matrix of the traditional QO-STBC system is discussed. The code structure adopted has been discussed in [8] following the QO-STBC method of code construction discussed in [6]. An interference free QOSTBC system achieves full diversity (see [11]) and the decoding matrix should be of the Equation 15 form [2, 13]. Equation 14 can be obtained as in $[2,8]$ by formulating:

$$
D=\left[\begin{array}{cccc}
\lambda+\beta & 0 & 0 & 0 \\
0 & \lambda+\beta & 0 & 0 \\
0 & 0 & \lambda-\beta & 0 \\
0 & 0 & 0 & \lambda-\beta
\end{array}\right]
$$

This can be obtained as in $[2,8]$ by formulating:

$$
D_{4} \cdot V-V \cdot D=0
$$

Where $V$ is the eigenvector as: 


$$
V=\left[\begin{array}{cccc}
1 & 0 & -1 & 0 \\
0 & 1 & 0 & -1 \\
1 & 0 & 1 & 0 \\
0 & 1 & 0 & 1
\end{array}\right]
$$

From Equation 16,

Now the resulting virtual channel matrix would be;

$$
\begin{aligned}
H_{\text {new }} & =H_{v} \cdot V \\
& =\left[\begin{array}{cccc}
h_{1}+h_{3} & h_{2}+h_{4} & h_{3}-h_{1} & h_{4}-h_{2} \\
h_{2}^{*}+h_{4}^{*} & -h_{1}^{*}-h_{3}^{*} & h_{4}^{*}-h_{2}^{*} & h_{1}^{*}-h_{3}^{*} \\
h_{1}+h_{3} & h_{2}+h_{4} & h_{1}-h_{3} & h_{2}-h_{4} \\
h_{2}^{*}+h_{4}^{*} & -h_{1}^{*}-h_{3}^{*} & h_{2}^{*}-h_{4}^{*} & h_{3}^{*}-h_{1}^{*}
\end{array}\right]
\end{aligned}
$$

Following the channel matrix of Equation 17 and the tradition in $[3,12]$, the encoding matrix can be formulated as:

$$
C_{\text {new }}=\left[\begin{array}{cccc}
c_{1}-c_{3} & c_{2}-c_{4} & c_{3}-c_{1} & c_{4}+c_{2} \\
c_{4}^{*}-c_{2}^{*} & -c_{3}^{*}+c_{1}^{*} & -c_{4}^{*}-c_{2}^{*} & c_{3}^{*}+c_{1}^{*} \\
c_{1}-c_{3} & c_{4}+c_{2} & c_{3}-c_{1} & c_{4}-c_{2} \\
-c_{4}^{*}-c_{2}^{*} & c_{3}^{*}+c_{1}^{*} & c_{4}^{*}-c_{2}^{*} & -c_{3}^{*}+c_{1}^{*}
\end{array}\right]
$$

By deleting the fourth column of the new matrix, the new matrix for a 3x1 QO-STBC can be formed thus;

$$
\left[\begin{array}{ccc}
c_{1}-c_{3} & c_{2}-c_{4} & c_{3}+c_{1} \\
c_{4}^{*}-c_{2}^{*} & -c_{3}^{*}+c_{1}^{*} & -c_{4}^{*}-c_{2}^{*} \\
c_{1}+c_{3} & c_{2}+c_{4} & c_{3}-c_{1} \\
-c_{4}^{*}-c_{2}^{*} & -c_{3}^{*}+c_{1}^{*} & c_{4}^{*}-c_{2}^{*}
\end{array}\right]
$$

By nulling the fourth antenna elements of the channel matrix in Equation 8, then the equivalent channel matrix for $3 \times 1$ can be formed.

$$
H_{\text {new3 }}=\left[\begin{array}{cccc}
h_{1}+h_{3} & h_{2} & h_{3}-h_{1} & -h_{2} \\
h_{2}^{*} & -h_{1}^{*}-h_{3}^{*} & -h_{2}^{*} & h_{1}^{*}-h_{3}^{*} \\
h_{1}+h_{3} & h_{2} & h_{1}-h_{3} & h_{2} \\
h_{2}^{*} & -h_{1}^{*}-h_{3}^{*} & h_{2}^{*} & h_{3}^{*}-h_{1}^{*}
\end{array}\right]
$$

As it would be expected, the $4 \times 1$ QO-STBC outperforms the $3 \times$ 1QO-STBC as shown in Figure 1.

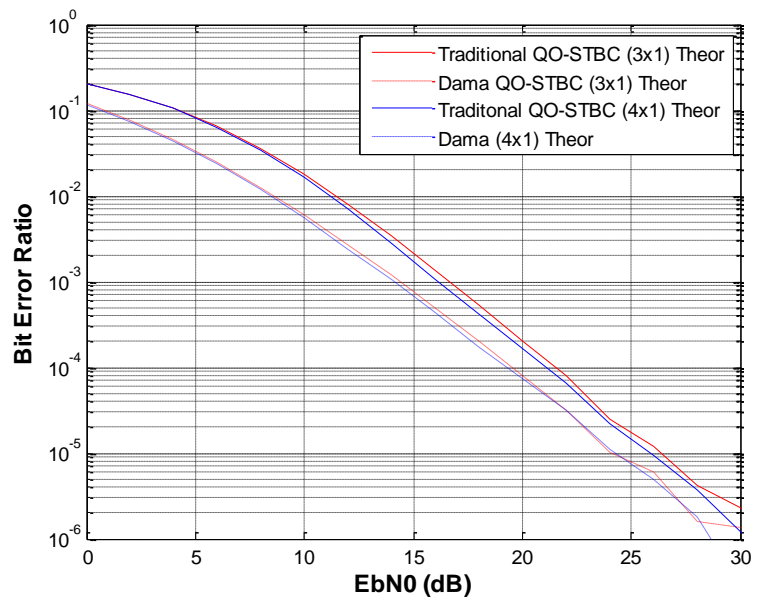

Fig 1. Comparison of Traditional QO-STBC and Interference-free QOSTBC

It is observed that interference elimination approach achieves circa $2 \mathrm{~dB}$ gain with respect to the traditional QOSTBC for both $4 \times 1$ QO-STBC and $3 \times 1$ QO-STBC. This approach is then extended to include the OFDM as discussed in the next section.

\section{The Qo-Stbc Ofdm System Model}

Combining the QO-STBC scheme and the OFDM system presented in Section II, the QO-STBC OFDM architecture is represented in Figure 2. The combination of QO-STBC scheme with OFDM drives the system towards achieving maximum diversity gain since in achieving maximum diversity gain, the number of subcarriers, $N$, must be larger than or equal to the number of independent delay paths, $L$, [11].

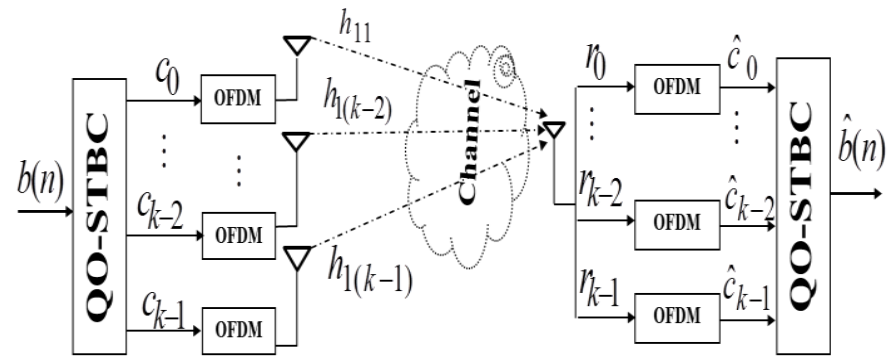

Fig 2. Design Architecture for QO-STBC OFDM System

This architecture is shown for a QPSK system with $b(n)$ as the resulting QPSK mapped symbols in the transmitter. Its encoding is according to the descriptions of the QO-STBC above. According to the number of the required transmit antennas, the output QO-STBC coded symbols $C_{0}, \ldots, C_{k-2}$, $C_{k-1}$ (where $k$ is the maximum number of transmit antennas) are individually modulated by the OFDM modulation scheme. After traversing the channel the OFDM symbols are received 
as $r_{0}, \cdots, r_{k-2}, r_{k-1}$ corresponding to each transmission branch. Then, the estimates are demodulated by the OFDM of each transmission branch to obtain the estimates of the QOSTBC encoded symbols, $\hat{c}_{0}, \cdots, \hat{c}_{k-2}, \hat{c}_{k-1}$, for onward QOSTBC decoding. These estimates of the OFDM modulated symbols are obtained by performing an $N$-point inverse Fourier transform (IFFT) and removing the CP. After decoding, an estimate of the transmitted symbol, $\hat{b}(n)$, is obtained. This is fed to the QPSK de-mapping block and then for error computation.

\section{Numerical Simulation RESUlts AND Discussion}

In the simulation, the symbols are constructed according to the codes. The codes provide, for three transmit antenna system, three time slots. So, the QO-STBC OFDM system symbols will be quasi-static for three slots. Also, for four time slots where no column of the encoding matrix is eliminated, then the QO-STBC OFDM symbols will be quasi-static for four time slots. The OFDM was designed with a CP length of $25 \%$. Recall that one of the major advantages of OFDM system is that it converts a frequency selective transmission channel to a non-frequency selective channel such that correlation difference of one channel impulse response to another is highly negligible. In addition to this advantage, this study investigated a scenario where the channel is necessarily correlated. This condition is described for a frequency nonselective transmission. Thus, the flat fading channel has the characteristics that the channel coefficients are strictly uniform for each transmission branch. The results in Figure 3 are identical in behaviour to the results earlier reported in Figure 1. It is shown that for all cases the QPSK QO-STBC interference-free OFDM systems outperformed the traditional QPSK QO-STBC OFDM systems. Notice that the interference free method is designated in Figure 3 after the name of the proposer, Dama et al.

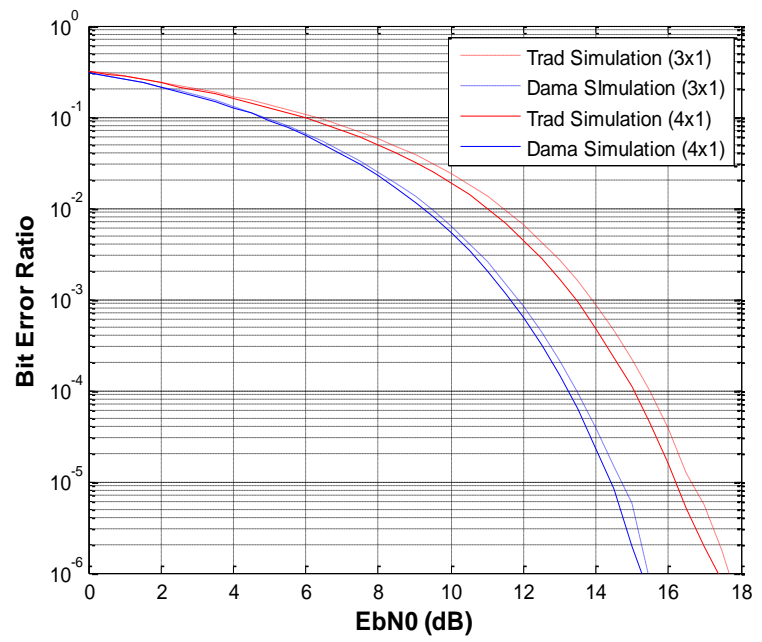

Fig 3. Comparison of Simulated results of interference QO-STBC OFDM system with traditional QO-STBC OFDM system

It is necessary to emphasize that the OFDM is characterized with better performance than the theoretical result. In QO-STBC OFDM, diversity advantage improves with the number of subcarrier. Also, the presence of cyclic prefix provides a phenomenon that reduces the irreducible error that is not possible in the traditional systems. In that sense, the inter-symbol interference are well overcome.

\section{CONCLUSION}

QO-STBC scheme that improve the performance of multi antenna system using diversity over OFDM system has been presented. Traditional QO-STBC systems achieve only full transmission rate but not full diversity. The full diversity limitation has been shown to be consequent on the interfering terms incurred during decoding. These interfering terms exist as off-diagonal elements in the decoding matrix, which when there is no interference the matrix is a diagonal matrix and so permit linear decoding. An interference elimination technique was used in the case of a QO-STBC OFDM system to improve the performance of the QO-STBC scheme over an OFDM system. The decoding matrix of the QO-STBC is constructed for a free-interference decoding which improves the system performance. It was observed that the scheme achieved about $2 \mathrm{~dB}$ gain both in theory and when OFDM system was applied during simulation. Consequently, the interference elimination method must be considered in the deployment of QO-STBC for multi antenna transmission in OFDM systems.

\section{ACKNOWLEDGMENT}

This work was partially sponsored by Datong plc, UK as part of an on-going research project and to be employed as part of their future communication services and financially by the Ebonyi State Government of Nigeria. The authors wish to thank the Ebonyi State Government for their support.

\section{REFERENCES}

[1] H. Jafarkhani, "A quasi-orthogonal space-time block code," IEEE Transactions on Communications, vol. 49, pp. 1-4, 2001.

[2] Y. A. S. Dama, R. A. Abd-Alhameed, S. M. R. Jones, H. S. O. Migdadi, and P. S. Excell, "A NEW APPROACH TO QUASI-ORTHOGONAL SPACE-TIME BLOCK CODING APPLIED TO QUADRUPLE MIMO TRANSMIT ANTENNAS," Fourth International Conference on Internet Technologies \& Applications, Sept. 2011, 2011.

[3] A. Goldsmith, Wireless communications: Cambridge university press, 2005.

[4] S. M. Alamouti, "A simple transmit diversity technique for wireless communications," Selected Areas in IEEE Journal on Communications, vol. 16, pp. 1451-1458, 1998.

[5] C. B. Papadias and G. J. Foschini, "Capacity-approaching space-time codes for systems employing four transmitter antennas," IEEE Transactions on Information Theory, vol. 49, pp. 726-732, 2003.

[6] O. Tirkkonen, A. Boariu, and A. Hottinen, "Minimal non-orthogonality rate 1 space-time block code for 3+ Tx antennas," in 2000 IEEE Sixth International Symposium on Spread Spectrum Techniques and Applications, 2000, pp. 429-432.

[7] W. Su and X.-G. Xia, "Signal constellations for quasi-orthogonal spacetime block codes with full diversity," IEEE Transactions on Information Theory, vol. 50, pp. 2331-2347, 2004.

[8] Y. Dama, R. Abd-Alhameed, T. Ghazaany, and S. Zhu, "A New Approach for OSTBC and QOSTBC," International Journal of Computer Applications, vol. 67, pp. 45-48, 2013.

[9] K. O. O. Anoh, R. A. Abd-alhameed, J. M. Noras, and S. M. R. Jones, "Wavelet Packet Transform Modulation for Multiple Input Multiple Output Applications," IJCA, vol. 63 - Number 7, pp. 46 - 51, 2013.

[10] D. Tse and P. Viswanath, Fundamentals of wireless communication: Cambridge university press, 2005. 
[11] F. Fazel and H. Jafarkhani, "Quasi-orthogonal space-frequency and space-time-frequency block codes for MIMO OFDM channels," IEEE Transactions on Wireless Communications, vol. 7, pp. 184-192, 2008.

[12] J. Proakis and M. Salehi, Digital Communications, Fifth ed. Asia: McGraw-Hill, 2008.
[13] U. Park, S. Kim, K. Lim, and J. Li, "A novel QO-STBC scheme with linear decoding for three and four transmit antennas," IEEE Communications Letters, vol. 12, pp. 868-870, 2008. 